\title{
Knowledge Is Safety: The Time Is Ripe for Hepatitis E Virus Blood Donor Screening
}

\author{
Tanja Vollmer Cornelius Knabbe Jens Dreier \\ Institut für Laboratoriums- und Transfusionsmedizin, Herz- und Diabeteszentrum Nordrhein-Westfalen, \\ Universitätsklinik der Ruhr-Universität Bochum, Bad Oeynhausen, Germany
}

Recently, Hewitt et al. [1] demonstrated transmission of hepatitis $\mathrm{E}$ viruses (HEV) via contaminated blood in $42 \%$ of transfusion recipients. The authors reported a prevalence rate of 1:2,848 HEV RNA-positive donors in England, a figure comparable to other European countries (Germany 1:1,240 [2], The Netherlands 1:1,761 [3]). In industrial countries, chronic, severe, or fatal HEV infections have been observed in immunosuppressed individuals (e.g. transplant patients, hematological patients [4]) and in individuals with pre-existing liver diseases $[5,6]$.

Which options do we have today to avoid HEV infection in high-risk transfusion recipients? First, vaccination is supposed to be one possibility, but so far not feasible because of the lack of an approved vaccine. At least one prophylactic vaccine against genotype 1 strains has been tested in a phase III clinical trial [7], but its efficacy for genotype 3 infections remains unknown. Second, identification of HEV-positive blood donors by donor screening will significantly reduce the risk of transfusion-transmitted infections. Several historic reasons in favor for blood donor screening for viral infections also apply to hepatitis $\mathrm{E}$ :

- Confirmed transmission by blood products in immunosuppressed patients [1].

- High HEV RNA prevalence in blood donors [1-3].

- A long viremic phase (4-6 weeks with titers up to $10^{5} \mathrm{IU} / \mathrm{ml}$ ) with or without variant clinical symptoms [8].

- Ineffective viral inactivation or reduction $[9,10]$.

- Higher mortality and morbidity in immunosuppressed patients [4].

These reasons do not necessarily result in HEV screening. Other viruses such as CMV and PVB19 infections are frequently in blood donors, and they are known to cause severe progression in at-risk patients. However, the current guidelines state against a general NAT testing for these viruses. Therefore, the crucial question arise, if $\mathrm{HEV}$ should be considered being in line with $\mathrm{CMV} /$ PVB19 or rather with HBV. In our opinion, the relative risk of transmission of HEV is exceedingly higher than for CMV/PVB19, even if the actual medical threat remains lower [11]. We strongly believe that the real clinical importance is still unidentified because the rate of reported transfusion-transmitted HEV infections is too small compared to the estimated incidence. Underestimation is caused by several factors, such as failure to recognize or misinterpretation of symptoms [12] or presentation of HEV infection much later than during the immediate post-transfusion period so that no association with an earlier transfusion is recognized [1]. Although HEV infections tend to present with subclinical or asymptomatic courses, the risk of serious complications including death (especially in immunocompromised individuals, i.e., the largest group of transfusion recipients) should not be neglected. This is supported by the work by Feray et al. [13] who concluded that transfusion of blood products not screened for HEV RNA is associated with the risk of chronic infection in immunocompromised patients. Certainly, the rate of transfusion transmissions does not tell anything about clinical importance. Since the infectious dose is not yet known, the incidence of HEV infection must not necessarily correlate with the number of transfusion-transmitted infections. However, data showing or excluding a clear evidence regarding the real clinical importance is missing. Additionally, one has to keep in mind that most HEV infections were transmitted via the zoonotic or food-borne route, and transmission of HEV infection via contaminated blood represents a subordinate role. Therefore, it is counterproductive to make only the blood safe for at-risk patients while at the same time other infection routes continue to infect those patients. The patient's benefit of HEV-RNA-negative blood components will depend on adapted dietary habits to reduce the risk of HEV infection by contaminated food. Indeed, this situation, complicating the assessment of HEV seen from the angle of blood safety, is partially comparable with that when regarding HIV. It is indisputable that the clinical implications of HIV infection are more severe than those so far known for hepatitis E, but the main risk of HIV transmission is also situated outside the transfusion medicine. Nonetheless

\section{KARGER}

(c) 2016 S. Karger GmbH, Freiburg

Fax +497614520714 
Fig. 1. Progression of hepatitis E viremia in seven blood donors with autochthonous HEV genotype 3 infection (modified according to Vollmer et. al [8]). Displayed is the course of HEV-RNA concentration. The day of the detection of HEV-RNA by PCR screening was defined as day 0 [2]. The HEV virus titer in positive plasma samples was quantified using the first WHO international standard for hepatitis E virus RNA for NAT-based assays (Paul Ehrlich Institute, Langen, Germany). The connecting lines for donors 6 and 7 are discontinuous due to a 40 -day lack of plasma samples. The greyshaded background covers the areas of positive donation detected by NAT (pool and ID-NAT) depending on different LODs (95\% LOD vs. 50\% LOD pool-NAT) $[2,18]$. The grey-shaded graph represents the course of the extrapolated idealized $\mathrm{HEV}$ viremia.

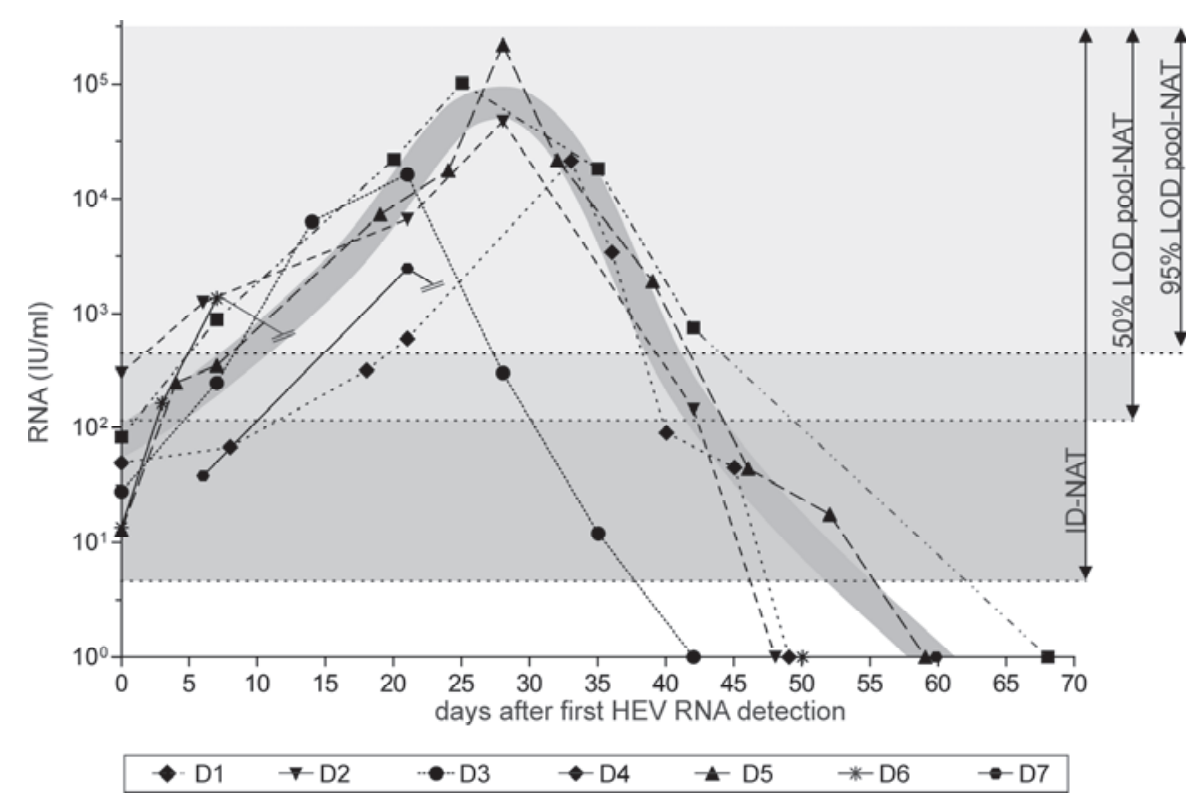

enormous efforts regarding HIV-NAT testing are carried out (e.g. dual-target-NAT [14]) to further minimize the already low residual infection risk. Very sensitive antigen-antibody assays are available, and the diagnostic window is shortened by NAT testing to only 2 days [15].

To date, still too little is known about key facts of transfusionassociated HEV infection since the infectious dose, the role of anti$\mathrm{HEV}$ in the recipient, predisposing factors, the differences between individuals experiencing asymptomatic or symptomatic infection, or the efficacy of inactivation are unsettled issues. To further elucidate at least one of these questions, we examined the progression of $\mathrm{HEV}$ viremia in plasma samples of $7 \mathrm{HEV}$ genotype 3-infected blood donors identified by routine HEV-RNA blood donor screening $[2,8]$.

Knowledge on the progression of viremia in blood donors provides the basis for the development of adequate screening strategies. We and others have previously shown that the diagnostic gap between a high viral load and serological confirmation of infection calls for NAT screening methods instead of serological approaches in order to effectively improve blood safety $[2,16]$. Nonetheless, the window phase in general also demands for NAT screening, not least due to the fact that HEV infection in blood donors often proceed asymptomatically and the lack of symptoms prevented the identification of viremic donors. But what would be the most useful screening sensitivity? The study by Hewitt et al. [1] revealed that an infective dose below approximately $500 \mathrm{IU} / \mathrm{ml}$ did not result in infection and that packed red blood cells (PRBCs) did not seem to be particularly important for HEV transmission. Therefore, the residual plasma volume of the transfused blood product appears to play an important role for the transmission of HEV infection. This brings up the question of how to correctly estimate the infective dose, which should be calculated in reference to the volume of transfused IU per blood product rather than as the viral concentration measured in the donor (IU/ml). Obviously, the immune status of the recipient has a major impact on the actual risk of infection caused by contaminated blood products, but all in all the infective dose remains undetermined today.

If all this mentioned above is taken into account, we also believe that HEV RNA screening is currently the only option and should be implemented in regions where HEV is endemic, according to Pawlotsky [11] and others. In contrast to Hewitt et al. [1], we do think that 'there is pressing need to move rapidly with the introduction of donation screening'. It is not surprising that the need for HEV blood donation screening is extensively discussed by several European committees. Factors such as the high frequency of viremic donors and the currently unknown incidence of overt transfusion-transmitted hepatitis E contrasting the limited chronicity to a small proportion of recipients, the primary moderate nature of HEV infection, and the related costs for NAT screening were taken into consideration. A prominent example for the progress towards introduction of HEV NAT screening is the implementation of a HEV run control for screening human plasma pools requested by the European Pharmacopoeia [17]. But what are the major issues for blood donation screening? Preferably, the most simple and rapid solution would be to add HEV screening to the current routine screening procedures. Most often, blood donation services perform minipool screening of up to 96 samples, but concerns about the required sensitivity remain. We introduced routine HEV NAT screening (96 minipool, 95\% LOD 4.7 IU/ml (95\% CI 3.6-7.6 IU/ml, $452 \mathrm{IU} / \mathrm{ml}$ per single donation [2])) for therapeutic blood products in our transfusion facility in January 2015. This strategy aims to identify highly viremic donors and will cover at least a large part of viremic phases. Taken into account the observed progression of viremia in 7 genotype 3 -infected blood donors (fig. 1), our implemented NAT screening method will detect $51.3 \%$ (95\%LOD) or $71.8 \%$ (50\% LOD) of viremic donations. However, it remains to be seen in the future if this strategy will detect all relevant viremic phases that could result in transfusiontransmitted hepatitis E. Unfortunately, some commercially available as well as in-house assays currently does not meet this require- 
ments, and an adequate reduction of the minipool size would be necessary to meet our consecutively proposed strategy. Additionally, ID testing is also an option, but increases costs and creates great demands on technical requirements. Alternative screening strategies to a general HEV screening may also be considered, e.g. screening of blood products for at-risk patients (immunosuppressed patients, pregnant women, or newborns). This approach is probably more cost-effective in any case but requires logistical implications; therefore, we adhere to our general screening strategy. Since the introduction of routine HEV RNA screening, we detected 76 HEV RNA-positive blood donors up to now with viremias ranging from $13.4-1.54 \times 10^{5} \mathrm{IU} / \mathrm{ml}$. The distribution of viral loads were as follows: $<100 \mathrm{IU} / \mathrm{ml}: \mathrm{n}=13,100-500 \mathrm{IU} / \mathrm{ml}: \mathrm{n}=20$, 500$1,000 \mathrm{IU} / \mathrm{ml}: \mathrm{n}=5,1,000-10,000 \mathrm{IU} / \mathrm{ml}: \mathrm{n}=14,>10,000 \mathrm{IU} / \mathrm{ml}: \mathrm{n}=$ 14 , not quantified: $\mathrm{n}=10$ ). Altogether, we still detect 33 donors with viremias below the $95 \%$ detection limit of assay by pool screening. In our opinion, the time is ripe for HEV screening in order to improve blood safety.

\section{Disclosure Statement}

The manuscript has been seen and approved by all authors, and we state that we have no conflict of interest.

\section{References}

1 Hewitt PE, Ijaz S, Brailsford SR, Brett R, Dicks S, Haywood B, Kennedy IT, Kitchen A, Patel P, Poh J, Russell K, Tettmar KI, Tossell J, Ushiro-Lumb I, Tedder RS: Hepatitis E virus in blood components: a prevalence and transmission study in southeast England. Lancet 2014;384:1766-1773.

2 Vollmer T, Diekmann J, Johne R, Eberhardt M, Knabbe C, Dreier J: Novel approach for the detection of hepatitis E virus infection in German blood donors. J Clin Microbiol 2012;50:2708-2713.

3 Hogema BM, Molier M, Slot E, Zaaijer HL: Past and present of hepatitis $\mathrm{E}$ in the Netherlands. Transfusion 2014;54:3092-3096.

4 Kamar N, Dalton HR, Abravanel F, Izopet J: Hepatitis E virus infection. Clin Microbiol Rev 2014;27:116-138.

$\checkmark 5$ Dalton HR, Hazeldine S, Banks M, Ijaz S, Bendall R: Locally acquired hepatitis $\mathrm{E}$ in chronic liver disease. Lancet 2007;369:1260.

6 Peron JM, Bureau C, Poirson H, Mansuy JM, Alric L Selves J, Dupuis E, Izopet J, Vinel JP: Fulminant liver failure from acute autochthonous hepatitis $\mathrm{E}$ in France: description of seven patients with acute hepatitis $\mathrm{E}$ and encephalopathy. J Viral Hepat 2007; 14:298-303.

7 Zhao Q, Zhang J, Wu T, Li SW, Ng MH, Xia NS, Shih JW: Antigenic determinants of hepatitis $\mathrm{E}$ virus and vaccine-induced immunogenicity and efficacy. J Gastroenterol 2013;48:159-168.
Vollmer T, Diekmann J, Eberhardt M, Knabbe C, Dreier J: Hepatitis E in blood donors: investigation of the natural course of asymptomatic infection, Germany 2011. Euro Surveill 2016;21: doi: 10.2807/15607917.

9 Andonov A, Rock G, Lin L, Borlang J, Hooper J, Grudeski E, Wu J: Serological and molecular evidence of a plausible transmission of hepatitis E virus through pooled plasma. Vox Sang 2014;107:213-219.

10 Hauser L, Roque-Afonso AM, Beyloune A, Simonet M, Fischer BD, des Roziers NB, Mallet V, Tiberghien P, Bierling P: Hepatitis E transmission by transfusion of intercept blood system-treated plasma. Blood 2014; 123:796-797.

11 Pawlotsky JM: Hepatitis E screening for blood donations: an urgent need? Lancet 2014;384:1729-1730.

12 Haim-Boukobza S, Ferey MP, Vetillard AL, Jeblaoui A, Pelissier E, Pelletier G, Teillet L, Roque-Afonso AM: Transfusion-transmitted hepatitis $\mathrm{E}$ in a misleading context of autoimmunity and drug-induced toxicity. J Hepatol 2012;57:1374-1378.

13 Feray C, Pawlotsky JM, Roque-Afonso AM, Samuel D, Dhumeaux D: Should we screen blood products for hepatitis E virus RNA? Lancet 2014;383:218.
4 Chudy M, Kress J, Halbauer J, Heiden M, Funk MB, Nübling CM: Risk minimization measures for blood screening HIV-1 nucleic acid amplification technique assays in Germany. Transfus Med Hemother 2014;41: 45-51.

15 Kleinman SH, Lelie N, Busch MP: Infectivity of human immunodeficiency virus- 1 , hepatitis $C$ virus, and hepatitis $\mathrm{B}$ virus and risk of transmission by transfusion. Transfusion 2009;49:2454-2489.

16 Baylis SA, Gartner T, Nick S, Ovemyr J, Blumel J: Occurrence of hepatitis $\mathrm{E}$ virus RNA in plasma donations from Sweden, Germany and the United States. Vox Sang 2012;103:89-90.

17 European Pharmacopoeia: Human plasma (pooled and treated for virus inactivation). European Pharmacopoeia 5.0 2014;01/2005:1646. http://library.njucm.edu.cn/ yaodian/ep/EP501E/16_monographs/17_monographs_ d-k/human_plasma_for_fractionation/0853e.pdf (last accessed October 13, 2016)

18 Vollmer T, Knabbe C, Dreier J: Comparison of realtime PCR and antigen assays for detection of hepatitis E virus in blood donors. J Clin Microbiol 2014;52: 2150-2156. 